\title{
Interpolation method for crystals with many-body interactions
}

\author{
Lukiya A. Mistryukova, ${ }^{1}$ Nikita P. Kryuchkov, ${ }^{1}$ Vladimir N. Mantsevich, ${ }^{1}$ \\ Andrei V. Sapelkin, ${ }^{2}$ and Stanislav O. Yurchenko ${ }^{1, *}$ \\ ${ }^{1}$ Bauman Moscow State Technical University, 2nd Baumanskaya street 5, 105005 Moscow, Russia \\ ${ }^{2}$ School of Physics and Astronomy, Queen Mary University of London, London E1 4NS, United Kingdom
}

(Dated: July 12, 2021)

\begin{abstract}
We propose an interpolation scheme to describe pair correlations in crystals with many-body interactions that requires only information on relative displacements for the nearest-neighbours and in the long range. Using crystalline $\mathrm{Ni}$ as a test case, the scheme is shown to deliver the functional form for the radial distribution function at least as well as molecular dynamics simulations. The results provide a fast route for verification of interatomic potentials and study of many-body interactions using a combination of x-ray scattering and x-ray absorption spectroscopy.
\end{abstract}

Many-body interactions feature widely in nature ${ }^{1}$, being intrinsic to solids ${ }^{2}$ and liquids ${ }^{3}$, metals, proteins, macromolecular, molecular, and colloidal systems ${ }^{4}$. Short and long-range interatomic correlations are intrinsically linked to these interactions (and the corresponding potentials), and hence are connected with the thermodynamic and elastic properties, activation phenomena, and a variety of excitations. However, the effect of manybody interactions on the fundamental properties of condensed matter remains largely unstudied. This is, not in the least, due to a challenging problem of developing a methodology that can provide direct experimental access to the many-body interactions and correlations in solids and liquids on atomic scale.

Dynamic processes in real atomic systems can be modelled using, for example, molecular dynamics (MD) simulations, but the latter require appropriate interatomic potentials in order to yield sufficiently reliable data. The choice of a potential is typically tested indirectly against experimentally-accessible properties such as heat capacity, bulk modulus, elastic constants, phonon dispersion spectra ${ }^{5}$. In . In some of the systems, most notably in molecules and nanostructures, access to the bulk properties is challenging if at all possible. As a consequence, the details of a variety of microscopic processes generated in MD simulations may be inaccurate or lost altogether. Experimental techniques such as neutron and x-ray scattering 10 can yield pair distribution function (PDF) and hence do provide direct access to the atomic structure and interatomic correlations, and thus to the information on the nature of interatomic potentials. However, when PDF data alone are used in solving the corresponding inverse problem of extracting information about potentials, the well-known ambiguities arise 15 with distinctly different interatomic interactions resulting in similar (or identical within experimental errors) PDFs.

At the same time, spectroscopic methods such as extended x-ray absorption fine structure (EXAFS) are particularly sensitive to the local interatomic correlations $5 \frac{18}{24}$ (and hence to the local interactions) and can provide information complementary to the scattering techniques. Indeed, these two methods are intrinsi- cally sensitive to the atomic dynamics at different length scales, for they are based on the near- (EXAFS) and farfield (PDF) x-ray interference effects, providing access to the short-range (EXAFS) and long-range (PDF) correlations. However, combination of these techniques is not generally utilised for the purpose of study and verification of interatomic potentials. One of the key issues is absence of the common correlation analysis framework for joint data interpretation. Furthermore, a numerous reports on comparison of the MD simulations and of the EXAFS-derived data all show as yet unexplained discrepancies $25 \sqrt{27}$ between experimental data and MDbased models. Besides, the ability to accurately account for distance-dependence of interatomic displacements in EXAF ${ }^{20}$ has not yet been addressed and discussions are still ongoing 28 . These discrepancies significantly limit application of EXAFS in recovering structural information and application of advanced methods (e.g. machine learning $29 \sqrt{31}$ ) and in analysis of complex data sets obtained, for example, under in-operando catalysis conditions $52 \mid 33$, in biology $\sqrt{34}$ and in complex novel nanostructures 35,37 .

Thus, the field of condensed matter research would benefit significantly from a method for fast PDF calculations and an analysis framework that should connect information contained in EXAFS and PDF data to the interatomic potentials. In the present article, we introduce just such a framework based on the Interpolation Method (IM, 38 411) extended to include many-body interactions found in real atomic systems. We describe a methodology for testing interatomic potentials by $d i$ rect comparison with the atomic-scale interactions and demonstrate that our approach can be utilised for fast and accurate calculations of PDF in real crystals.

Pair correlations and many-body interactions. - Consider a crystal of particles with pairwise and three-body forces. At thermodynamic equilibrium in the canonical (NVT) ensemble, the pair and three-body correlation functions should minimise the Helmholtz free energy of the crystal, $F=F_{2}+F_{3}$, where $F_{2,3}=E_{2,3}-T S_{2,3}$ correspond to pairwise and three-body interactions (as denoted by the subscripts), $E$ and $S$ are the interaction energy and the entropy, and $T$ is the temperature (we 
use energy units). Similarly to Ref ${ }^{\sqrt[40]{0}}$, using the expansion for entropy through correlation functions reported in Ref ${ }^{42}$, we obtain

$$
\begin{aligned}
& F_{2}=\frac{n^{2}}{2} \int d \mathbf{r}_{1} d \mathbf{r}_{2} g_{2}(1,2)\left[\varphi(1,2)+T \ln g_{2}(1,2)\right], \\
& F_{3}=\frac{n^{3}}{6} \int d \mathbf{r}_{1} d \mathbf{r}_{2} d \mathbf{r}_{3} g_{3}(1,2,3) \times \\
& \times\left[\varphi_{3}(1,2,3)+T \ln \widetilde{g}_{3}(1,2,3)\right],
\end{aligned}
$$

where $n=N / V$ is the particle density, $N$ is the total number of particles in the volume $V, g_{2}(1,2) \equiv g_{2}\left(\mathbf{r}_{1}, \mathbf{r}_{2}\right)$ and $g_{3}(1,2,3) \equiv g_{3}\left(\mathbf{r}_{1}, \mathbf{r}_{2}, \mathbf{r}_{3}\right)$ are the pair and threebody correlation functions, the same abbreviations of arguments we use for pairwise and three-body interaction potentials, $\varphi(1,2)$ and $\varphi_{3}(1,2,3) ; \widetilde{g}_{3}(1,2,3)=$ $g_{3}(1,2,3) / g_{2}(1,2) g_{2}(1,3) g_{2}(2,3)$ and we assume that $\widetilde{g}_{3}(1,2,3) \rightarrow 1$ at large distances between the particles.

Since the particles in a crystal move near their equilibrium positions, we can write ${ }^{40}$

$$
g(\mathbf{r})=\frac{1}{n} \sum_{\alpha} p_{\alpha}\left(\mathbf{r}-\mathbf{r}_{\alpha}\right)
$$

where $g(\mathbf{r}) \equiv g_{2}\left(\mathbf{r}_{2}-\mathbf{r}_{1}\right)$, and each function $p_{\alpha}(\mathbf{r})$ is a spatial probability density, describing the contribution by a particle $\alpha$ with equilibrium position $\mathbf{r}_{\alpha}$. Since the peaks are localised spatially, we can consider the following moments

$$
\begin{aligned}
& \int d \mathbf{r} p_{\alpha}(\mathbf{r})=1, \quad \int d \mathbf{r} \mathbf{r} p_{\alpha}(\mathbf{r})=0 \\
& \int d \mathbf{r}\left(\mathbf{e}_{\alpha} \cdot \mathbf{r}\right)^{2} p_{\alpha}(\mathbf{r})=\sigma_{\| \alpha}^{2}, \\
& \int d \mathbf{r}\left(\mathbf{r}^{2}-\left(\mathbf{e}_{\alpha} \cdot \mathbf{r}\right)^{2}\right) p_{\alpha}(\mathbf{r})=(D-1) \sigma_{\perp \alpha}^{2}, \\
& \int d \mathbf{r}\left(\mathbf{e}_{\alpha} \cdot \mathbf{r}\right)^{3} p_{\alpha}(\mathbf{r})=\gamma_{\alpha}
\end{aligned}
$$

where $\mathbf{e}_{\alpha}=\mathbf{r}_{\alpha} /\left|\mathbf{r}_{\alpha}\right|$ is a unit vector in the $\mathbf{r}_{\alpha}$-direction, $D=3$ is the spatial dimension, $\sigma_{\| \alpha}^{2}$ and $\sigma_{\perp \alpha}^{2}$ are the longitudinal and transverse components of the MSRD of the particle $\alpha$, and $\gamma_{\alpha}$ is the third moment in radial direction. The first two conditions in (3) are related to the normalisation per particle and equilibrium position of a particle at node $\alpha$.

Now, to find the functional form of pair correlation peaks, we minimise the functional of the Helmholtz free energy $F\left\{p_{\alpha}, \widetilde{g}_{3}\right\}$ and obtain $\widetilde{g}_{3}=\exp \left(-\varphi_{3} / T\right)$ and

$$
\begin{aligned}
p_{\alpha}(\mathbf{r}) & =C_{\alpha} \exp \left[-\frac{\varphi\left(\mathbf{r}+\mathbf{r}_{\alpha}\right)}{T}-b_{\alpha}\left(\mathbf{e}_{\alpha} \cdot \mathbf{r}\right)-\right. \\
& \left.-\frac{\left(\mathbf{e}_{\alpha} \cdot \mathbf{r}\right)^{2}}{2 a_{\| \alpha}^{2}}-\frac{\mathbf{r}^{2}-\left(\mathbf{e}_{\alpha} \cdot \mathbf{r}\right)^{2}}{2 a_{\perp \alpha}^{2}}-d_{\alpha}\left(\mathbf{e}_{\alpha} \cdot \mathbf{r}\right)^{3}\right],
\end{aligned}
$$

(see Supplementary Materials ${ }^{43}$ ) where the constants $C_{\alpha}$, $a_{\| \alpha}^{2}, a_{\perp \alpha}^{2}, b_{\alpha}$, and $d_{\alpha}$ are determined by Eqs. (3). Note that (4) was derived directly from (1)-(3), using the variation free energy.

We see that the profile (4) has the same functional form as if we assumed the interactions to be pairwise. In this approximation, three-body interactions just affect the second and third moments in (3). In the same manner one can consider many-body interactions of higher orders (using expansions for entropy reported in $\frac{42}{2}$ ), to illustrate that they do not change the functional form (4). Therefore, having the temperature dependencies of $\sigma_{\|, \perp \alpha}^{2}$ and $\gamma_{\alpha}$ for different particles $\alpha$, one can obtain $g(\mathbf{r})$ at different temperatures for a given crystalline lattice and pairwise potential $\varphi(r)$.

The number of free parameters within the outlined theoretical description can be significantly reduced using the interpolation method (IM). Within IM, if we walk between sites through the shortest graph on the crystalline lattice, the MSRDs are growing recurrently (in 3D case) with each step as 39

$$
\widetilde{\sigma}_{\alpha+1}^{2}=\widetilde{\sigma}_{\alpha}^{2}+1-2 \phi \sqrt{\widetilde{\sigma}_{\alpha}^{2}},
$$

where $\alpha$ denotes the number of steps in the shortest graph, $\widetilde{\sigma}_{\alpha}^{2}=\sigma_{\alpha}^{2} / \sigma_{1}^{2}$, and $\phi=\sqrt{\sigma_{1}^{2} / \sigma_{\infty}^{2}} / 2$.

We can see that the method described above interpolates between the two correlation regimes - from short distances where local correlations are significant to large distances where at the limit of $\sigma_{\infty}^{2}$ the relative correlations vanish - all the while taking into account the functional form of individual correlation peaks (4). Thus, using the IM we can reconstruct the correlation peaks $p_{\alpha}(\mathbf{r})$ if we know $\sigma_{\|, \perp 1}^{2}$ (for the nearest neighbours) and $\sigma_{\infty}^{2}$. In the case of the expression (4) modified to include the three-body interactions, we additionally need to know $\gamma$ parameter for the nearest particles, and a posteriori analysis proves high accuracy of such approach. One of the key elements of our approach is that parameters $\sigma_{\| 1}^{2}$ and $\gamma$ can be obtained experimentally with EXAFS, whereas the (isotropic) function $g(r)=\int d \Omega g(\mathbf{r}) / 4 \pi$ (here, $\Omega$ is the solid angle) can be measured with x-ray (or neutron) scattering and used to extract $\sigma_{\infty}^{2}$.

In what follows we demonstrate that EXAFS data can be used to identify the most suitable embedded-atom model potential (EAM) for MD simulations. We then use MD simulations to obtain the required IM parameters across the wide range from room temperature to the melting point. Finally, we demonstrate that the modified IM delivers excellent agreement with the MD simulations and experimental PDF data for crystalline Ni. We use crystalline $\mathrm{Ni}$ as a test case on account of well-known many-body affects in metals ${ }^{44}$ and availability of the experimental data.

Results and discussion. - First we tested the ability of EXAFS data to discriminate different EAM models used in MD simulations to reproduce the temperature behaviour of $\sigma_{\|}^{2}$ and $\gamma$ in $\mathrm{Ni}$, since a number of reports exist all showing discrepancies $25 \sqrt{27}$ between experimental data and MD-based models. The details of the MD 

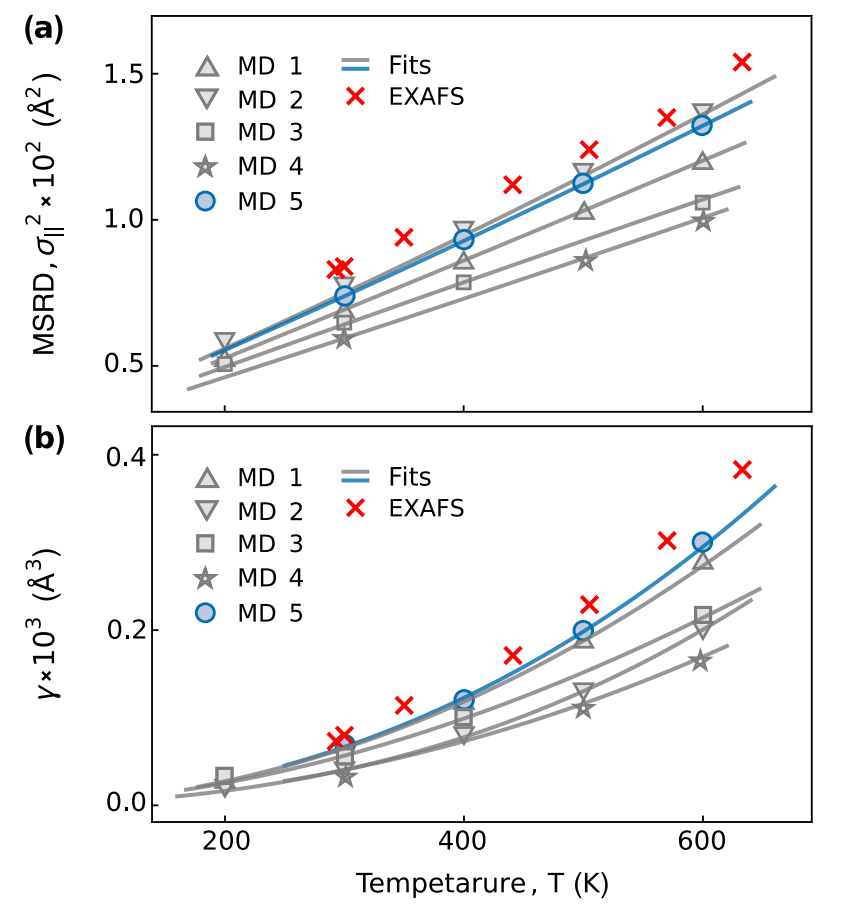

FIG. 1. The parameters of the first correlation peak obtained with EXAFS and MD simulations: The moments (a) $\sigma_{\| 1}^{2}$ and (b) $\gamma$, corresponding to the first correlation peak, obtained with EXAFS (red crosses), MD simulations (grey and blue symbols) for fcc Ni lattice at different temperatures. The solid grey and blue lines are fits (6).

simulations, EXAFS data collection, and analysis can be found in Supplemental Materials $\$ 33$. We considered five EAM models developed for crystalline Ni (models MD $1^{9}$, $\mathrm{MD}^{5}, \mathrm{MD}^{5}{ }^{6}, \mathrm{MD}^{5}$ ) ) and for $\mathrm{Ni}$ glass (MD2 ${ }^{8}$ ).

The comparison of experimental EXAFS data and MD results is provided in Fig. 1. Here, the experimental results are shown with red crosses, whereas MD results are shown with grey symbols for the models MD1-MD4 and with blue circles for the model MD5. The solid grey and blue lines are the following fits

$$
\sigma_{\| 1}^{2}=\alpha_{1, \|} T\left(1+\beta_{1, \|} T\right), \quad \gamma=\alpha_{\gamma} T^{2}\left(1+\beta_{\gamma} T\right)
$$

where $\alpha$ and $\beta$ are fitting parameters. The coefficients $\alpha_{1, \|}$ and $\beta_{1, \|}$ describe the temperature-dependence of MSRDs and their anharmonic corrections, respectively. We see that the agreement between MD data and EXAFS displays an unexpected sensitivity to the exact parameters of EAM potentials and, hence, a capability do discriminate between a selection of EAM models. Based on the data in Fig. 1, we conclude that the model MD5 provides the best description of both microscopic parameters $\sigma_{\|}^{2}$ and $\gamma$ for the first correlation peak (4). Therefore, we used the model MD5 for further analysis of pair correlation functions.

Having verified the most appropriate potential model for $\mathrm{Ni}$, we utilised $\mathrm{MD}$ simulations to obtain $\sigma_{1}^{2}, \sigma_{\infty}^{2}$,

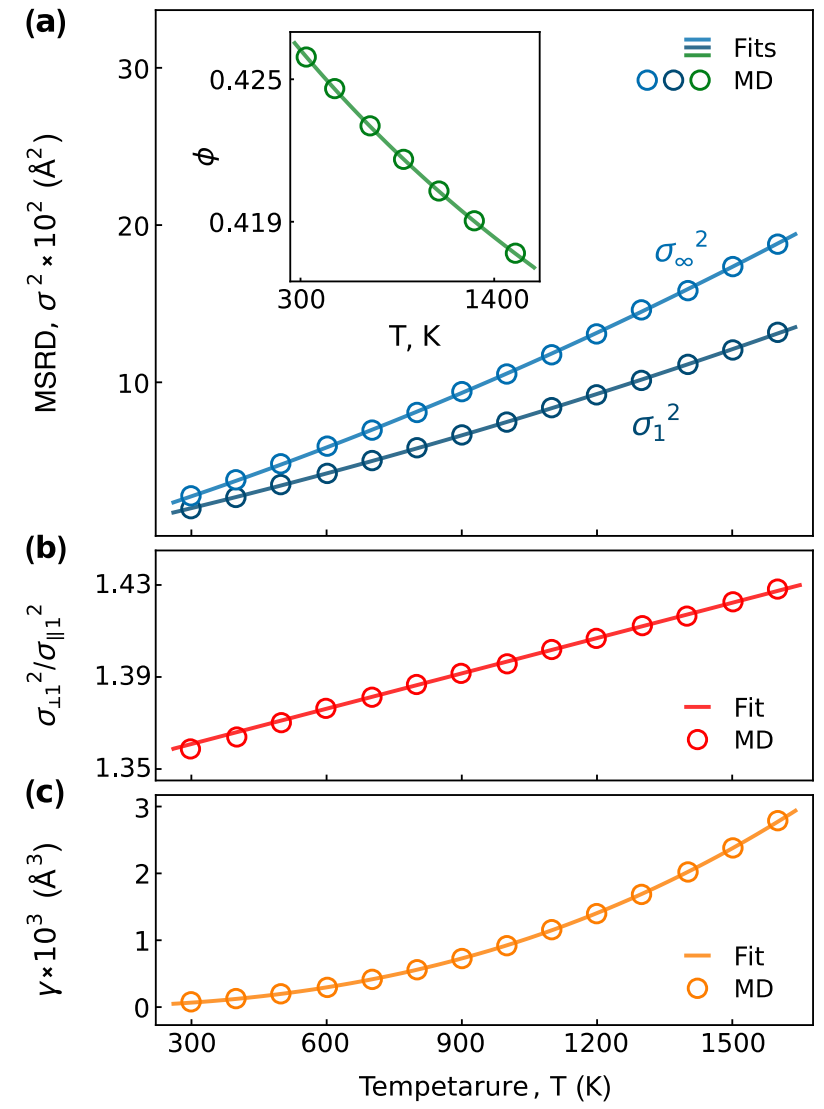

FIG. 2. The temperature evolution of parameters characterising fcc Ni lattice: (a) the MSRDs $\sigma_{1}^{2}$ (light blue symbols) and $\sigma_{\infty}^{2}$ (dark blue symbols), the correlation parameter (green symbols), and approximations with fits (7) (solid lines). (b) The asymmetry coefficient $\sigma_{\perp 1}^{2} / \sigma_{\| 1}^{2}$ (red circles) and its fit (red solid line). (c) The $\gamma$-parameter for the first correlation peak (circles) and fit (6) (solid line). The density was taken under normal conditions.

the asymmetric coefficient $\sigma_{\perp 1}^{2} / \sigma_{\| 1}^{2}$, and the third moment $\gamma$ of the first peak along the axis $\mathbf{e}_{\alpha}$, throughout the extended temperature range from room to melting temperature, Fig. 2. The symbols correspond to the MD results, the fits used here for MSRDs are

$$
\begin{aligned}
& \sigma_{1, \infty}^{2}=\alpha_{1, \infty} T\left(1+\beta_{1, \infty} T\right), \\
& \sigma_{\perp 1}^{2} / \sigma_{\| 1}^{2}=\alpha_{a} T\left(1+\beta_{a} T\right) .
\end{aligned}
$$

The values of the coefficients $\alpha_{1, \infty}, \beta_{1, \infty}$ we obtained for fcc Ni are presented in the Table I in Suppl. Materials 43 . We see that using only two pairs of $\sigma_{1}^{2}$ and $\sigma_{\infty}^{2}$ (at high and low temperatures) one can reconstruct the MSRDs dependencies across the entire temperature range, from the cold crystal to the melting line. We can now also derive the correlation parameter $\phi$ using Eq. (5) (see inset in Fig. 2(a) and observe (Fig. 2(b)) that the asymmetry between the transverse and longitudinal MSRDs $\sigma_{\perp 1}^{2} / \sigma_{\| 1}^{2}$ increases linearly with temperature growth. The radial third moment $\gamma$ increases with temperature too, as ex- 

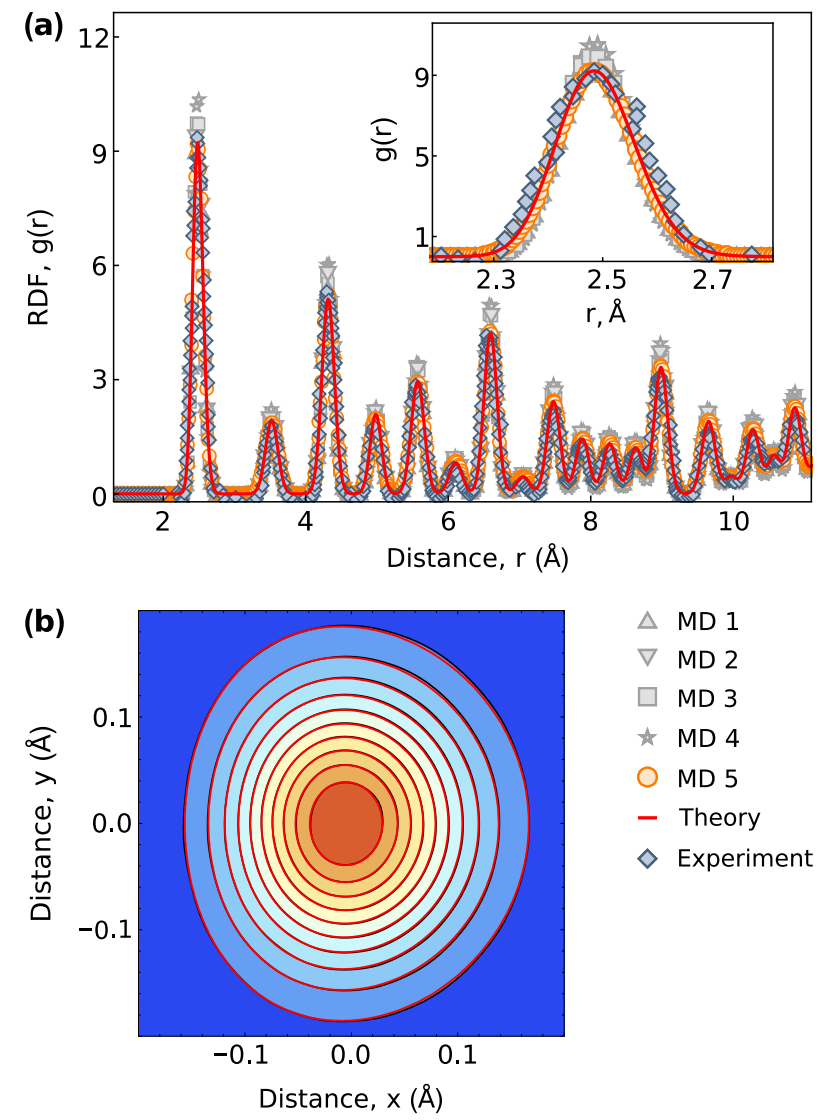

FIG. 3. Pair correlations in crystalline Ni: (a) The radial distribution function for $3 \mathrm{D}$ fcc Ni lattice at $T=300$. The diffraction data is presented by red symbols, the model MD5 is marked by orange circles and the other models are marked by gray symbols. Solid lines are related to the theoretical curves recieved from the IM. (b) The first peaks in color-code format at the same temperature calculated using the MD5 model. Red lines correspond to the isolines of theoretical fit with IM.

pected (Fig. 2(c)). With the results for MSRDs and third moments, we can now calculate $g(r)$ with the IM.

Finally, we compare $g(r)$ at $T=300 \mathrm{~K}$ obtained with the IM and experimentally 10 (see Fig. 3(a)). Here, the MD results for MD5 model and experimental data are marked by blue diamonds and orange circles, respectively. The models MD1-MD4 are also presented here by the same grey symbols as in the Fig. 11. Solid red line is the theoretical profile obtained with the IM using the results shown in Figs. 1 and 2 . The insets show a closeup of the first correlation peak. The results perfectly illustrate the ambiguities described in the introduction: all potentials (MD1-MD5) used in MD simulations result in a rather good agreement with the experimental PDF data. This further emphasises importance of utilising EXAFS data for verification of the interatomic potentials. Crucially, numerical analysis shows that the IM theory (solid red line) shows the best agreement with the experimental data together with the MD5 model verified by EXAFS (see Table II in Suppl. Materials ${ }^{43}$ ).

To further illustrate the versatility and the level of insight IM can provide into the atomic dynamics on microscopic scale, the $2 \mathrm{D}$ cross-section of the first correlation peak is shown in Fig. 3(b) as a colour-coded map. The red isolines here correspond to IM, and show excellent agreement with the black isolines of the MD5 result, demonstrating IM capabilities to recover the full microscopic picture of MSRDs. Overall, the model that provides the best description for $\sigma_{\| 1}^{2}$ and the third moment $\gamma$ also provides the best description of the pair correlation function $g(\mathbf{r})$. Note that the profile of the first correlation peak is excellently described with the parametric form (4). Three-body interactions just affect the temperature dependencies of the parameters of the correlation peaks, but not its functional form. These results clearly demonstrate that the IM provides precise connection between the short and long-range correlations, which can be studied experimentally with EXAFS and x-ray scattering techniques.

Conclusions. - Here, we developed an IM that takes into account three-body interactions. We found that in this implementation only normalization of the $\sigma_{\infty}^{2}$ and $\sigma_{1}^{2}$ dependencies is required: many-body interactions have shown to only renormalise parameters of pair correlation peaks, but not their functional form. We show that parameters required for PDF calculations using IM can be obtained from MD simulations. Crucially, we demonstrate that EXAFS can be used to guide a selection of the best suitable MD potential as it shows remarkable sensitivity to their specific form.

Combining experiments, MD simulations, and the developed IM approach, we studied face-centric cubic Ni crystal as a representative system. The validity of the new IM has been tested by comparing the results with experimental x-ray scattering data both in the long and short range order. Furthermore, we demonstrate that using IM, information only at two different temperatures (low value and close one to the melting point) is needed to recover atomic displacements over the entire temperature range. The main aim of our manuscript was to propose the IM for systems with many-body interactions, whereas the case of Ni was used a test. Study of more complicated cases (e.g., ionic crystals) should be performed in future.

In summary, we demonstrate that EXAFS shows remarkable ability to discriminate interatomic potentials through atomic displacements suggesting a new methodology for direct verification, or indeed development, of interactomic potentials. In combination with the new IM approach, our methodology describes the atomic dynamics on microscopic scale as well as MD simulations do. Due to the nature of the methodology, this approach can be adapted to a a wide selection of systems including molecules and nanoparticles. It is also clear that the modified IM can be used for analysis of many-body interactions and joint analysis x-ray scattering and EXAFS data. 


\section{ACKNOWLEDGMENTS}

L.A.M., N.P.K., and S.O.Y., acknowledge RFBR Grant No. 20-32-70186 (theory and analysis of pair cor- relations), V.N.M. acknowledges BMSTU State Assignment (infrastructural support); A.V.S. acknowledges the Royal Society International Exchanges IES $\backslash R 1 \backslash 191077$ grant.
* st.yurchenko@mail.ru

1 S. P. Veccham, J. Lee, and M. Head-Gordon, The Journal of Chemical Physics 151, 194101 (2019).

${ }^{2}$ D. V. Tuan, B. Scharf, Z. Wang, J. Shan, K. F. Mak, I. Žutić, and H. Dery, Physical Review B 99, 085301 (2019)

${ }^{3}$ G. R. Medders, A. W. Götz, M. A. Morales, P. Bajaj, and F. Paesani, The Journal of Chemical Physics 143, 104102 (2015)

4 J. Dobnikar, R. Rzehak, and H. H. von Grünberg, Europhysics Letters (EPL) 61, 695 (2003).

Y. Mishin, D. Farkas, M. J. Mehl, and D. A. Papaconstantopoulos, Physical Review B 59, 3393 (1999).

${ }^{6}$ G. J. Ackland, G. Tichy, V. Vitek, and M. W. Finnis, Philosophical Magazine A 56, 735 (1987)

7 X. W. Zhou, R. A. Johnson, and H. N. G. Wadley, Physical Review B 69, 144113 (2004)

M. Mendelev, M. Kramer, S. Hao, K. Ho, and C. Wang, Philosophical Magazine 92, 4454 (2012).

9 S. M. Foiles, M. I. Baskes, and M. S. Daw, Physical Review B 33, 7983 (1986)

${ }^{10}$ P. Juhás, T. Davis, C. L. Farrow, and S. J. L. Billinge, Journal of Applied Crystallography 46, 560 (2013)

11 M. J. Cliffe, M. T. Dove, D. A. Drabold, and A. L. Goodwin, Physical Review Letters 104, 125501 (2010).

12 I.-K. Jeong, R. H. Heffner, M. J. Graf, and S. J. L. Billinge, Physical Review B 67, 104301 (2003).

13 T. Proffen, S. J. L. Billinge, T. Egami, and D. Louca, Zeitschrift für Kristallographie - Crystalline Materials 218, 20664 (2003)

${ }^{14}$ P. Fornasini, S. a Beccara, G. Dalba, R. Grisenti, A. Sanson, M. Vaccari, and F. Rocca, Physical Review B 70, 174301 (2004)

Io H. Wang, F. H. Stillinger, and S. Torquato, The Journal of Chemical Physics 153, 124106 (2020).

${ }^{10}$ F. H. Stillinger and S. Torquato, The Journal of Chemical Physics 150, 204125 (2019)

${ }_{17}$ F. Frommer, M. Hanke, and S. Jansen, Journal of Mathematical Physics 60, 093303 (2019).

18 A. V. Sapelkin, S. C. Bayliss, A. G. Lyapin, V. V. Brazhkin, and A. J. Dent, Physical Review B 56, 11531 (1997)

I9 A. V. Poiarkova and J. J. Rehr, Physical Review B 59, 948 (1999)

20 A. V. Sapelkin and S. C. Bayliss, Physical Review B 65, $172104(2002)$

${ }^{21}$ G. Dalba, P. Fornasini, F. Rocca, and S. Mobilio, Physical Review B 41, 9668 (1990).

${ }_{22}$ A. Filipponi and P. D'Angelo, in X-Ray Absorption and X-Ray Emission Spectroscopy (John Wiley \& Sons, Ltd, 2016) pp. 745-771.

23 A. D. Cicco, F. Iesari, A. Trapananti, P. D'Angelo, and A. Filipponi, The Journal of Chemical Physics 148, 094307 (2018)
${ }^{24}$ E. C. O'Quinn, K. E. Sickafus, R. C. Ewing, G. Baldinozzi, J. C. Neuefeind, M. G. Tucker, A. F. Fuentes, D. Drey, and M. K. Lang, Science Advances 6, eabc2758 (2020)

25 J. Timoshenko, A. Kuzmin, and J. Purans, Open Physics 9, $710(2011)$.

20 S. W. T. Price, N. Zonias, C.-K. Skylaris, T. I. Hyde, B. Ravel, and A. E. Russell, Physical Review B 85, 075439 (2012)

27 V. Migliorati, A. Serva, G. Aquilanti, L. Olivi, S. Pascarelli, O. Mathon, and P. D'Angelo, Physical Chemistry Chemical Physics 17, 2464 (2015)

${ }_{28}$ P. Fornasini and R. Grisenti, Journal of Synchrotron Radiation 22, 1242 (2015).

${ }^{29}$ J. Terry, M. L. Lau, J. Sun, C. Xu, B. Hendricks, J. Kise, M. Lnu, S. Bagade, S. Shah, P. Makhijani, A. Karantha, T. Boltz, M. Oellien, M. Adas, S. Argamon, M. Long, and D. P. Guillen, Applied Surface Science 547, 149059 (2021).

30 J. Timoshenko, D. Lu, Y. Lin, and A. I. Frenkel, The Journal of Physical Chemistry Letters 8, 5091 (2017)

${ }^{31}$ C. Zheng, K. Mathew, C. Chen, Y. Chen, H. Tang, A. Dozier, J. J. Kas, F. D. Vila, J. J. Rehr, L. F. J. Piper, K. A. Persson, and S. P. Ong, npj Computational Materials 4, $12(2018)$.

${ }^{32}$ E. Bus, J. T. Miller, A. J. Kropf, R. Prins, and J. A. van Bokhoven, Physical Chemistry Chemical Physics 8, 3248 (2006)

33 Z. Wei, J. Sun, Y. Li, A. K. Datye, and Y. Wang, Chemical Society Reviews 41, 7994 (2012).

${ }^{34}$ W. Meyer-Klaucke, M. Gnida, and G. Henkel, in Reference Module in Chemistry, Molecular Sciences and Chemical Engineering (Elsevier, 2014).

35 B. Zhang, T. Zhu, M. Ou, N. Rowell, H. Fan, J. Han, L. Tan, M. T. Dove, Y. Ren, X. Zuo, S. Han, J. Zeng, and K. Yu, Nature Communications 9, 2499 (2018)

${ }^{36}$ Y. Liu, L. Tan, G. Cibin, D. Gianolio, S. Han, K. Yu, M. T. Dove, and A. V. Sapelkin, Nanoscale 12, 19325 (2020).

37 L. Tan, A. J. Misquitta, A. Sapelkin, L. Fang, R. M. Wilson, D. S. Keeble, B. Zhang, T. Zhu, F. S. Riehle, S. Han, K. Yu, and M. T. Dove, Nanoscale 11, 21900 (2019).

38 S. O. Yurchenko, The Journal of Chemical Physics 140, $134502(2014)$

39 S. O. Yurchenko, N. P. Kryuchkov, and A. V. Ivlev, The Journal of Chemical Physics 143, 034506 (2015)

40 S. O. Yurchenko, N. P. Kryuchkov, and A. V. Ivlev, Journal of Physics: Condensed Matter 28, 235401 (2016)

${ }_{41}$ N. P. Kryuchkov, S. O. Yurchenko, Y. D. Fomin, E. N. Tsiok, and V. N. Ryzhov, Soft Matter 14, 2152 (2018).

42 D. C. Wallace, The Journal of Chemical Physics 87, 2282 (1987)

43 See Supporting Information (URL).

44 M. S. Daw, S. M. Foiles, and M. I. Baskes, Materials Science Reports 9, 251 (1993) 